\title{
Флуктуации интенсивности в слабонелинейном случайном сигнале в оптоволокне с накачкой
}

\author{
С.С. Вергелес $^{1,2, *}$, Л.Л. Огородников ${ }^{1,3}$ \\ ${ }^{1}$ Институт теоретической физики им. Л.Д. Ландау РАН, Черноголовка, Московская область \\ ${ }^{2}$ Физический факультет Высшей школь экономики, Москва \\ ${ }^{3}$ Сколковский институт научки и технологии, Сколково \\ *E-mail: ssver@itp.ac.ru
}

DOI:10.31868/RFL2018.70-71

Сигнал в оптоволокне часто имеет нерегулярную форму, как, например, в сверхдлинных ВКР-лазерах [1,2] или в закодированном потоке данных. Даже если на входе в оптоволокно локальные статистические свойства такого сигнала имеют специфические особенности, то после прохождения многих дисперсионных длин эти свойства становятся универсальным. Если эволюция сигнала строго линейна и определяется хроматической дисперсией, то статистика сигнала приближается к гауссовой статистике [3]. В частности, вероятность сильных но редких всплесков интенсивности сигнала определяются хвостом функции распределения интенсивности, который по мере распространения всё более приближается к распределению Релея.

При увеличении средней интенсивности сигнала начинает играть роль нелинейность, изменяя свойства универсального статистического предела. Для фокусирующего нелинейного уравнения Шредингера нелинейность увеличивает вероятность редких всплесков интенсивности, тогда как для дефокусирующего уменьшает [4].

В представляемой работе [5] мы исследовали одноточечную статистику редких всплесков интенсивности в режиме слабой нелинейности, когда дисперсионная длина $1 / \beta \Delta^{2}$ мала по сравнению с нелинейной длиной $1 / \gamma\langle I\rangle$, где $\langle I\rangle-$ средняя интенсивность сигнала, $\gamma$ - коэффициент керровской нелинейности, $\beta-$ коэффициент хроматической дисперсии, $\Delta$ - ширина спектра сигнала. Мы предполагали, что к моменту измерения сигнал успел пройти много дисперсионных длин. Мы ограничились рассмотрением всплесков не очень большой амплитуды $I$, удовлетворяющей условию $\quad I /\langle I\rangle \ll$ $\sqrt{\beta \Delta^{2} / \gamma\langle I\rangle}$. Статистику таких всплесков можно исследовать аналитически методом теории возмущений, вычисляя слабое отклонение статистики сиг-

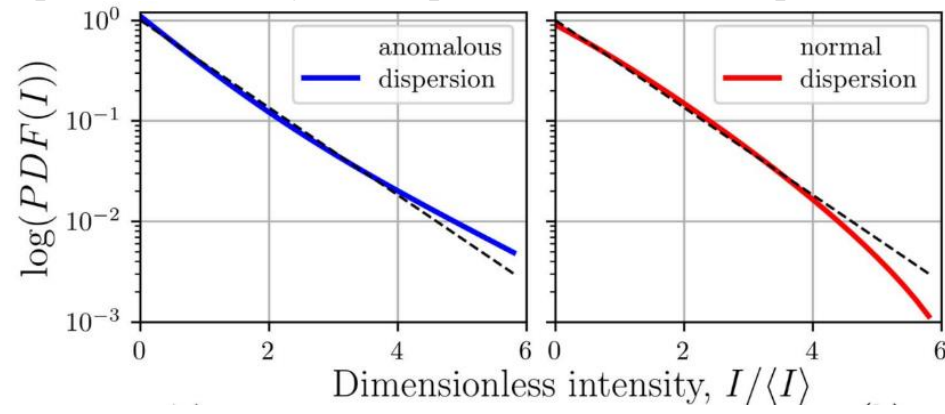

Рис. 1 PDF для интенсивности в случаях фокусирующего и дефокусирующего НУШ. нала от гауссовой вследствие действия нелинейности [6]. Непосредственно вычислялась безразмерная поправка к среднему значению квадрата интенсивности $K=\left\langle I^{2}\right\rangle /\langle I\rangle^{2}-2$, которая может быть названа отклонением куртозиса от его значения для гауссовой статистики. По величине $K$ можно восстановить функцию распределения в первом порядке по слабой нелинейности, см. Рис. 1.

В отсутствии накачки выражение для величины $K$ есть: 


$$
K_{0}=-\frac{\gamma\langle I\rangle}{\beta \Delta^{2}} \int \frac{\mathrm{d} \omega}{\pi} f(\omega) \tilde{f}^{2}(\omega),
$$

где спектр сигнала $F(\omega)=\langle I\rangle f(\omega)$, безразмерный спектр удовлетворяет условию нормировки $\int(\mathrm{d} \omega / 2 \pi) f(\omega)=1$, центрирован так что $\int \omega \mathrm{d} \omega f(\omega)=0$, а ширина спектра есть $\Delta^{2}=\int \omega^{2}(\mathrm{~d} \omega / 2 \pi) f(\omega)=1$. Кроме того, преобразование Гильберта от спектра обозначено как $\tilde{f}(\omega)=\int\left(\mathrm{d} \omega^{\prime} / \pi\right) f\left(\omega+\omega^{\prime}\right) / \omega^{\prime}$. Это же выражение в ранее было получено в [7].

Если в линейном режиме распространения сигнала усиление не влияет на статистику относительной интенсивности $I /\langle I\rangle$ всплесков, то в нелинейном режиме величина коэффициента усиления $g$ изменяет эту статистику. При слабом коэффициенте усиления, когда $g / \beta \Delta^{2} \ll$ 1 , воздействие нелинейности на статистические свойства сигнала набирается на последней (перед точкой измерения) дисперсионной длиной $\sim 1 / \beta \Delta^{2}$. Если же длина усиления становится малой по сравнению с дисперсионной длиной, так что $g / \beta \Delta^{2} \ll 1$, то нелинейность наиболее эффективно действует только на последней длине $\sim 1 / g$. В этом пределе отклонение куртозиса становится пропорциональным длине усиления, так что

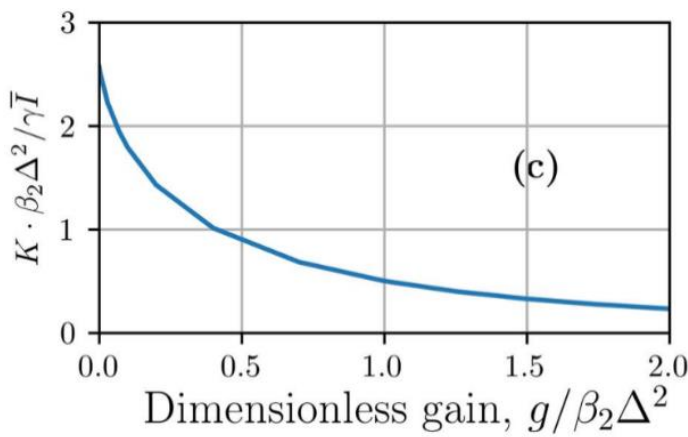

Рис. 2 Зависимость $K$ от коэффициента усиления $g$ $K \sim\left(\beta \Delta^{2} / g\right) K_{0}$. Численно полученная нами зависимость изображена на Рис. 2.

Наконец, нами было получено точное соотношение, связывающее между собой ширину спектра и статистику флуктуаций интенсивности, для нелинейного сигнала распространяющегося в среде с усилением (потерями):

$$
\frac{d}{d z} K=-\frac{\beta}{\gamma\langle I\rangle} \frac{d}{d z} \Delta^{2}
$$

Это соотношение является обобщением соотношения для чистого НУШ [8] и может быть использовано для верификации численного счёта.

Работа была поддержана грантом РФФИ 17-02-00929 А.

\section{Литература}

[1] Turitsyn, S. K., et al, Physics Reports, 542(2), 133-193 (2014)

[2] D.V. Churkin, et al, Nature Communications, vol. 6, p. 6214 (2015)

[3] Vergeles, S., \& Turitsyn, S. K. Physical Review A, 83(6), 061801 (2011)

[4] Agafontsev D., et al, XXVI Научная сессия Совета PAH по нелинейной динамике, дек. 2017.

[5] Ogorodnikov, L. L., and S. S. Vergeles. Optics letters 43 651-654 (2018)

[6] Kolokolov I.V., et al, ЖЭТФ, т. 146, c. 1295 (2014)

[7] Janssen, P. A. Journal of Physical Oceanography, 33(4), 863-884 (2003).

[8] Onorato, et al, Physics Letters A, 380(39), 3173-3177 (2016). 